\title{
A Shifted-Inverse Adaptive Multigrid Method for the Elastic Eigenvalue Problem
}

\author{
Bo Gong ${ }^{1}$, Jiayu $\mathrm{Han}^{2, *}$, Jiguang Sun ${ }^{3}$ and Zhimin Zhang ${ }^{1,4}$ \\ ${ }^{1}$ Beijing Computational Science Research Center, Beijing 100193, China. \\ 2 School of Mathematical Sciences, Guizhou Normal University, Guiyang, 550025, \\ China. \\ ${ }^{3}$ Department of Mathematical Sciences, Michigan Technological University, Houghton, \\ MI 49931, USA. \\ ${ }^{4}$ Department of Mathematics, Wayne State University, Detroit, MI 48202, USA.
}

Received 4 November 2018; Accepted 5 January 2019

\begin{abstract}
A shifted-inverse iteration is proposed for the finite element discretization of the elastic eigenvalue problem. The method integrates the multigrid scheme and adaptive algorithm to achieve high efficiency and accuracy. Error estimates and optimal convergence for the proposed method are proved. Numerical examples show that the proposed method inherits the advantages of both ingredients and can compute low regularity eigenfunctions effectively.
\end{abstract}

AMS subject classifications: $65 \mathrm{~N} 25,65 \mathrm{~N} 30$

Key words: Elastic eigenvalue problem, shifted-inverse iteration, adaptive multigrid method.

\section{Introduction}

Numerical computation of eigenvalue problems is of fundamental importance in many scientific and engineering applications. Due to the flexibility in treating complex structures and rigorous theoretical justification, finite element methods, including conforming finite elements, mixed finite elements, and discontinuous Galerkin methods, have been popular for eigenvalue problems of partial differential equations $[1,2,20]$. In this paper, we focus on the development of an efficient finite element discretization for the elastic eigenvalue problem on polygonal domains, in particular, when the regularity of the eigenfunctions might be low due to the non-convex domains or discontinuous material properties.

While extensive results have been obtained for the Dirichlet eigenvalue problem, the Maxwell's eigenvalue problem, and the biharmonic eigenvalue problem, much fewer

*Corresponding author. Email addresses: gongbo@csrc.ac.cn (B. Gong), hanjiayu126@126.com (J. Han), jiguangs@mtu.edu (J. Sun), zmzhang@csrc.ac.cn (Z. Zhang) 
works exist in the literature on the elastic eigenvalue problem $[9,10,13,16,18,19,22]$. In [19], Ovtchinnikov and Xanthis employed a special preconditioning technique associated with the effective dimensional reduction algorithm for the thin elastic structures such as shells, plates and rods of arbitrary geometry. Walsh et al. developed an a posteriori error estimator for heterogeneous elastic structures, which are independent of the variations in material properties and the polynomial degree of finite elements [22]. In [13], Erwin analyzed the finite element approximation of the spectral problem for the linear elasticity equation with mixed boundary conditions on a curved non-convex domain. Using a mixed variational formulation, Meddahi et al. showed that the lowest order Arnold-Falk-Winther element provides a correct approximation of the spectrum with quasi-optimal error estimates [18]. In [9], Russo presented a theory for the approximation of eigenvalue problems in mixed form by non-conforming methods and applied it to the classical Hellinger-Reissner mixed formulation for a linear elastic structure. Recently, Lee et al. used the immersed finite element method based on Crouzeix-Raviart P1-nonconforming element to approximate eigenvalue problems for elasticity equations with interfaces [16]. Domínguez et al. [10] analyzed the Jone's eigenvalue problem, an elastic eigenvalue problem with a special boundary condition, and approximated the eigenvalues using the linear Lagrange element.

The multigrid or multilevel discretization is an efficient tool for eigenvalue problems $[11,14,17,23,24]$. Recently, it was used with the shifted-inverse iteration and proved to be effective for the Dirichlet eigenvalue problem [25,26], the Maxwell eigenvalue problem, and the integral operator eigenvalue problem [26]. In this paper, we extend the shifted-inverse iteration multigrid method for the elastic eigenvalue problem. To further improve the accuracy and efficiency for lower regularity problems, the adaptive scheme is integrated into the multigrid method. Numerical examples show that the proposed method is highly effective and efficient. For adaptive finite element approximation of eigenvalue problems, we refer the readers to Chp. 8 of $[20],[7,8,12,15]$ and the references therein.

The rest of the paper is organized as follows. In Section 2, we present the elastic eigenvalue problem and finite element convergence results. Section 3 is devoted to the multigrid scheme based on Rayleigh quotient iteration. The convergence results are proved. In Section 4, we present an a posterior error estimate and propose an adaptive method. The algorithm combining the multigrid scheme and the adaptive method is given. Finally, in Section 5, we present several numerical examples on a non-convex domain and of discontinuous material properties, validating the efficiency and accuracy of the proposed method.

\section{FEM for the elastic eigenvalue problem}

Let $\mathbf{x}=(x, y)^{\top} \in \mathbb{R}^{2}, \Omega \subset \mathbb{R}^{2}$ be a bounded Lipschitz polygon, and $\mathbf{H}_{0}^{1}(\Omega):=H_{0}^{1}(\Omega) \times H_{0}^{1}(\Omega)$, $\mathbf{L}^{2}(\Omega):=L^{2}(\Omega) \times L^{2}(\Omega)$. The elastic eigenvalue problem is to find $(\lambda, \mathbf{u}) \in \mathbb{R} \times \mathbf{H}_{0}^{1}(\Omega)$ such 
that

$$
\begin{aligned}
-\nabla \cdot \sigma(\mathbf{u})=\lambda \rho \mathbf{u}, & \text { in } \Omega, \\
\mathbf{u}=\mathbf{0}, & \text { on } \partial \Omega .
\end{aligned}
$$

Here $\mathbf{u}(\mathbf{x})=\left(u_{1}(\mathbf{x}), u_{2}(\mathbf{x})\right)^{\top}$ is the displacement vector, $\rho(\mathbf{x})$ is the mass density, and $\sigma(\mathbf{u})$ is the stress tensor given by the generalized Hooke law

$$
\sigma(\mathbf{u})=2 \bar{\mu} \varepsilon(\mathbf{u})+\bar{\lambda} \operatorname{tr}(\varepsilon(\mathbf{u})) I,
$$

where $I \in \mathbb{R}^{2 \times 2}$ is the identity matrix and $\bar{\mu}, \bar{\lambda}$ are the Lamé parameters satisfying $\bar{\mu}>0$, $\bar{\lambda}+\bar{\mu}>0$. The strain tensor $\varepsilon(\mathbf{u})$ is defined as

$$
\varepsilon(\mathbf{u})=\frac{1}{2}\left(\nabla \mathbf{u}+(\nabla \mathbf{u})^{\top}\right),
$$

where $\nabla \mathbf{u}$ is the displacement gradient tensor

$$
\nabla \mathbf{u}=\left[\begin{array}{ll}
\partial_{x} u_{1} & \partial_{y} u_{1} \\
\partial_{x} u_{2} & \partial_{y} u_{2}
\end{array}\right]
$$

The weak formulation for (2.1) can be written as to find $(\lambda, \mathbf{u}) \in \mathbb{R} \times \mathbf{H}_{0}^{1}(\Omega)$ with $\|\mathbf{u}\|_{a}=$ 1 such that

$$
a(\mathbf{u}, \mathbf{v})=\lambda b(\mathbf{u}, \mathbf{v}), \quad \forall \mathbf{v} \in \mathbf{H}_{0}^{1}(\Omega)
$$

where

$$
a(\mathbf{u}, \mathbf{v})=\int_{\Omega} \sigma(\mathbf{u}): \nabla \mathbf{v} d \mathbf{x}, \quad b(\mathbf{u}, \mathbf{v})=\int_{\Omega} \rho \mathbf{u v} d \mathbf{x},
$$

$\|\mathbf{u}\|_{a}=\sqrt{a(\mathbf{u}, \mathbf{u})}$ and $\|\mathbf{u}\|_{b}=\sqrt{b(\mathbf{u}, \mathbf{u})}$. Here $A: B=\operatorname{tr}\left(A B^{\top}\right)$ is the Frobenius inner product of matrices $A$ and $B$. Without loss of generality, we assume that $\rho \equiv 1$ in the rest of the paper. The results in this paper hold for $\rho(\mathbf{x}) \geq \beta>0$.

Consider the finite element approximation for the elastic eigenvalue problem. Let $\pi_{h}$ be a regular triangular mesh on $\Omega$ under the meaning of [5] and $U_{h}$ be the linear Lagrange finite element space $U_{h}=\left\{\mathbf{u}_{h} \in \mathbf{H}_{0}^{1}(\Omega) ;\left.\mathbf{u}_{h}\right|_{\kappa} \in \mathbf{P}_{1}(\kappa), \kappa \in \pi_{h}\right\}$. The finite element discretization of (2.2) is as follows. Find $\left(\lambda_{h}, \mathbf{u}_{h}\right) \in \mathbb{R} \times U_{h}$ with $\left\|\mathbf{u}_{h}\right\|_{a}=1$ such that

$$
a\left(\mathbf{u}_{h}, \mathbf{v}_{h}\right)=\lambda_{h}\left(\mathbf{u}_{h}, \mathbf{v}_{h}\right), \quad \forall \mathbf{v}_{h} \in U_{h} .
$$

The well-posedness of (2.2) and (2.3) has been discussed in [1,3]. Consequently, there exist two linear bounded operators $T: \mathbf{L}^{2}(\Omega) \rightarrow \mathbf{H}_{0}^{1}(\Omega) \subset \mathbf{L}^{2}(\Omega)$ such that

$$
a(T \mathbf{f}, \mathbf{v})=b(\mathbf{f}, \mathbf{v}), \quad \forall \mathbf{v} \in \mathbf{H}_{0}^{1}(\Omega),
$$


and $T_{h}: \mathbf{L}^{2}(\Omega) \rightarrow U_{h} \subset \mathbf{H}_{0}^{1}(\Omega)$ such that

$$
a\left(T_{h} \mathbf{f}, \mathbf{v}\right)=b(\mathbf{f}, \mathbf{v}), \quad \forall \mathbf{v} \in U_{h} .
$$

Suppose that $\lambda$ and $\lambda_{h}$ are the $k$ th eigenvalue of (2.2) and (2.3), respectively. The algebraic multiplicity of $\lambda$ is equal to $q$, i.e., $\lambda=\lambda_{k}=\lambda_{k+1}=\cdots=\lambda_{k+q-1}$. Let $M(\lambda)$ be the space spanned by all the eigenfunctions corresponding to $\lambda$ and $M_{h}(\lambda)$ be the direct sum of eigenfunctions corresponding to the $q$ eigenvalues of (2.3) that converge to $\lambda$. Let $\widehat{M}(\lambda)=\left\{\mathbf{v}: \mathbf{v} \in M(\lambda),\|\mathbf{v}\|_{a}=1\right\}$ and $\widehat{M}_{h}(\lambda)=\left\{\mathbf{v}: \mathbf{v} \in M_{h}(\lambda),\|\mathbf{v}\|_{a}=1\right\}$. The following quantity characterizes the approximation of the finite element space $U_{h}$ for the eigenspace $\widehat{M}(\lambda)$ :

$$
\delta_{h}=\delta_{h}(\lambda)=\sup _{\mathbf{u} \in \widehat{M}(\lambda)} \inf _{\mathbf{v} \in U_{h}}\|\mathbf{u}-\mathbf{v}\|_{a} .
$$

Then an a priori error estimate for (2.3) follows the classical finite element spectral approximation theory $[1,2,4,20]$.

Lemma 2.1. Let $\left(\lambda_{h}, \mathbf{u}_{h}\right)$ be the kth eigenpair of (2.3) with $\left\|\mathbf{u}_{h}\right\|_{a}=1$. Let $\lambda$ be the kth eigenvalue of (2.2). Then $\lambda_{h} \rightarrow \lambda$ as $h \rightarrow 0$ and there exists an eigenfunction $\mathbf{u}$ corresponding to $\lambda$ such that

$$
\begin{aligned}
& \left|\lambda_{h}-\lambda\right| \leq C \delta_{h}(\lambda)^{2}, \\
& \left\|\mathbf{u}_{h}-\mathbf{u}\right\|_{b} \leq C h^{r} \delta_{h}(\lambda), \\
& \left\|\mathbf{u}_{h}-\mathbf{u}\right\|_{a} \leq C \delta_{h}(\lambda),
\end{aligned}
$$

where $r \leq 1$ is positive depending on the regularity of the source problem associated to (2.1), C denotes a positive constant that is independent of the mesh size.

\section{Multigrid scheme based on Rayleigh quotient iteration}

In this section, we present a multigrid scheme based on Rayleigh quotient iteration. Let $\left\{U_{h_{i}}, i=0,1,2, \cdots\right\}$ be a family of finite-dimensional spaces such that $U_{H}:=U_{h_{0}}, U_{h_{i}} \subset U_{h_{i+1}}$. The following condition is needed for the subsequent analysis.

Condition 3.1. Let $I$ be a positive integer. There exists two small positive numbers $\varepsilon_{1}, \varepsilon_{2}$ and $t_{i} \in\left[1+\varepsilon_{1}, 3-\varepsilon_{2}\right], i=1,2, \cdots, I$ such that $\delta_{h_{i}}=\mathcal{O}\left(\delta_{h_{i-1}}^{t_{i}}\right)$.

Condition 3.1 is easy to satisfy. For example, for smooth solutions, using the uniform mesh, let $h_{0}=\sqrt{2} / 8, h_{1}=\sqrt{2} / 32$ and $h_{3}=\sqrt{2} / 128$. We have $h_{i}=h_{i-1}^{t_{i}}$, i.e., $\delta_{h_{i}}=\mathcal{O}\left(\delta_{h_{i-1}}^{t_{i}}\right)$, where $t_{1} \approx 1.80, t_{2} \approx 1.22, t_{3} \approx 1$.18. For non-smooth solutions, the condition is met on the local refined meshes generated by the standard adaptive procedure.

In this section, we extend the multigrid scheme in $[25,26]$ to the elastic eigenvalue problem and prove the error estimates.

Algorithm 3.1. Multigrid Scheme. 
1. Solve the eigenvalue problem (2.3) on $U_{H}$ : find $\left(\lambda_{H}, \mathbf{u}_{H}\right) \in \mathbb{R} \times U_{H},\left\|\mathbf{u}_{H}\right\|_{a}=1$ such that

$$
a\left(\mathbf{u}_{H}, \mathbf{v}\right)=\lambda_{H} b\left(\mathbf{u}_{H}, \mathbf{v}\right), \quad \forall \mathbf{v} \in U_{H}
$$

2. $\mathbf{u}^{h_{0}} \Leftarrow \mathbf{u}_{H}, \lambda^{h_{0}} \Leftarrow \lambda_{H}, i \Leftarrow 1$.

3. Solve a source problem on $U_{h_{i}}$ : find $\mathbf{u}^{\prime} \in U_{h_{i}}$ such that

$$
a\left(\mathbf{u}^{\prime}, \mathbf{v}\right)-\lambda^{h_{i-1}} b\left(\mathbf{u}^{\prime}, \mathbf{v}\right)=b\left(\mathbf{u}^{h_{i-1}}, \mathbf{v}\right), \quad \forall \mathbf{v} \in U_{h_{i}} .
$$

Set $\mathbf{u}^{h_{i}}=\mathbf{u}^{\prime} /\left\|\mathbf{u}^{\prime}\right\|_{a}$.

4. Compute the Rayleigh quotient

$$
\lambda^{h_{i}}=\frac{a\left(\mathbf{u}^{h_{i}}, \mathbf{u}^{h_{i}}\right)}{b\left(\mathbf{u}^{h_{i}}, \mathbf{u}^{h_{i}}\right)} .
$$

5. If $i=l$ where $l$ is the maximum iterative times, then output $\left(\lambda^{h_{i}}, \mathbf{u}^{h_{i}}\right)$, stop; else, $i \Leftarrow i+1$, and go to 3 .

Define

$$
\operatorname{dist}(\mathbf{w}, W)=\inf _{\mathbf{v} \in W}\|\mathbf{w}-\mathbf{v}\|_{a} .
$$

Let the finite element approximation for $\lambda_{k-1}, \cdots, \lambda_{k+q}$ be $\lambda_{k-1, h}, \cdots, \lambda_{k+q, h}$ respectively and write

$$
v:=1 / \lambda, \quad v_{j}:=\lambda_{j}^{-1}, \quad v_{j, h}:=\lambda_{j, h}^{-1}, \quad j=k-1, k \cdots, k+q .
$$

Denote $M_{h}(v):=M_{h}(\lambda)$. The following lemmas are useful in the error analysis for the multigrid scheme.

Lemma 3.1 (Lemma 3.1 of [25]). For any nonzero $\mathbf{u}, \mathbf{v} \in U_{h}$

$$
\left\|\frac{\mathbf{u}}{\|\mathbf{u}\|_{a}}-\frac{\mathbf{v}}{\|\mathbf{v}\|_{a}}\right\|_{a} \leq 2 \frac{\|\mathbf{u}-\mathbf{v}\|_{a}}{\|\mathbf{u}\|_{a}}, \quad\left\|\frac{\mathbf{u}}{\|\mathbf{u}\|_{a}}-\frac{\mathbf{v}}{\|\mathbf{v}\|_{a}}\right\|_{a} \leq 2 \frac{\|\mathbf{u}-\mathbf{v}\|_{a}}{\|\mathbf{v}\|_{a}}
$$

The next lemma is an analog to Lemma 4.1 of [26] and can be proved similarly.

Lemma 3.2. Let $\left(v_{0}, \mathbf{u}_{0}\right)$ be an approximate eigenpair of $(v, \mathbf{u})$, where $v_{0}$ is not an eigenvalue of $T_{h}$ and $\mathbf{u}_{0} \in U_{h}$ with $\left\|\mathbf{u}_{0}\right\|_{a}=1$. Suppose that

$$
\begin{aligned}
& \operatorname{dist}\left(\mathbf{u}_{0}, M_{h}(v)\right) \leq 1 / 2, \\
& \left|v_{0}-v\right| \leq d / 4, \\
& \left|v_{j, h}-v_{j}\right| \leq d / 4, \quad j=k-1, k, k+q, \quad j \neq 0,
\end{aligned}
$$


where $d=\min _{v_{j} \neq v}\left|v_{j}-v\right|$. Let $\mathbf{u}^{s} \in U_{h}$ and $\mathbf{u}^{h} \in U_{h}$ satisfy

$$
\left(v_{0}-T_{h}\right) \mathbf{u}^{s}=\mathbf{u}_{0}, \quad \mathbf{u}^{h}=\mathbf{u}^{s} /\left\|\mathbf{u}^{s}\right\|_{a} .
$$

Then

$$
\operatorname{dist}\left(\mathbf{u}^{h}, M_{h}(v)\right) \leq \frac{4}{d} \max _{k \leq j \leq k+q-1}\left|v_{0}-v_{j, h}\right| \operatorname{dist}\left(\mathbf{u}_{0}, M_{h}(v)\right) .
$$

Let $\lambda_{j, h_{l}}, j=k, k+1, \cdots, k+q-1$, be $q$ eigenvalues of $T_{h_{l}}$ converge to $\lambda$ and $\mathbf{u}_{j, h_{l}}$ 's be the associated eigenfunctions such that $\left\{\mathbf{u}_{j, h_{l}}\right\}_{j=k}^{k+q-1}$ is an orthonormal system of $M_{h_{l}}(\lambda)$ with respect to $\|\cdot\|_{a}$.

From Lemma 2.1, there exists $\left\{\mathbf{u}_{j}^{0}\right\}_{j=k}^{k+q-1} \subset M(\lambda)$ such that $\mathbf{u}_{j, h_{l}}-\mathbf{u}_{j}^{0}$ satisfies (2.6) and (2.7). Let

$$
\mathbf{u}=\sum_{j=k}^{k+q-1} a\left(\mathbf{u}^{h_{l}}, \mathbf{u}_{j, h_{l}}\right) \mathbf{u}_{j}^{0} .
$$

The following lemma holds (see Theorem 4.2 in [26]).

Lemma 3.3. Assume that Condition 3.1 holds, $\mathbf{u}^{h_{l-1}}$ approximates some $\overline{\mathbf{u}} \in \widehat{M}(\lambda)$ and $\lambda^{h_{l-1}}$ approximates $\lambda$. If $\left(\lambda^{h_{l}}, \mathbf{u}^{h_{l}}\right)$ is the kth eigenpair approximation of (2.2) obtained by Algorithm 3.1 , then there hold

$$
\begin{aligned}
& \left\|\mathbf{u}^{h_{l}}-\mathbf{u}\right\|_{a} \leq C\left(\left|\lambda^{h_{l-1}}-\lambda\right|^{2}+\left|\lambda^{h_{l-1}}-\lambda\right|\left\|\mathbf{u}^{h_{l-1}}-\overline{\mathbf{u}}\right\|_{a}+\delta_{h_{l}}\right), \\
& \left|\lambda^{h_{l}}-\lambda\right| \leq C\left\|\mathbf{u}^{h_{l}}-\mathbf{u}\right\|_{a^{\prime}}^{2}
\end{aligned}
$$

where $l \geq 1$ and $\mathbf{u}$ is defined as in (3.2), and $C$ is independent of the mesh size and $l$.

Now we are ready to carry out the error analysis for Algorithm 3.1.

Theorem 3.1. Suppose that $H$ is small enough and Condition 3.1 holds. Let $\left(\lambda^{h_{l}}, \mathbf{u}^{h_{l}}\right)$ be the kth eigenpair approximation of (2.2) obtained by Algorithm 3.1. Then there hold

$$
\begin{aligned}
& \left\|\mathbf{u}^{h_{l}}-\mathbf{u}\right\|_{a} \leq C \delta_{h_{l^{\prime}}} \\
& \left|\lambda^{h_{l}}-\lambda\right| \leq C \delta_{h_{l^{\prime}}}^{2}
\end{aligned}
$$

where $l \geq 1$ and $\mathbf{u}$ is defined by (3.2).

Proof. The proof uses mathematical induction. When $l=1$, noting that $\mathbf{u}^{h_{0}}=\mathbf{u}_{H}$ and $\lambda^{h_{0}}=\lambda_{H}$, by (2.5), (2.7) and (3.1), there exists $\overline{\mathbf{u}} \in \widehat{M}(\lambda)$ such that

$$
\begin{aligned}
& \left\|\mathbf{u}^{h_{0}}-\overline{\mathbf{u}}\right\|_{a} \leq C \delta_{h_{0}}, \\
& \left|\lambda^{h_{0}}-\lambda\right| \leq C \delta_{h_{0}}^{2} .
\end{aligned}
$$


Plugging the above two formulae into (3.3) and Condition 3.1 we obtain

$$
\left\|\mathbf{u}^{h_{1}}-\mathbf{u}\right\|_{a} \leq C\left(\delta_{h_{0}}^{4}+\delta_{h_{0}}^{3}+\delta_{h_{1}}\right) \leq C \delta_{h_{1}},
$$

where $\mathbf{u}$ is given by (3.2) with $\mathbf{u}_{j, h_{1}}-\mathbf{u}_{j}^{0}$ satisfying (2.6)-(2.7). Substitution of (3.7) into (3.4) yields

$$
\left|\lambda^{h_{1}}-\lambda\right| \leq C \delta_{h_{1}}^{2}
$$

Thus (3.5)-(3.6) hold for $l=1$.

Suppose (3.5)-(3.6) hold for $l-1$, i.e., there hold

$$
\begin{aligned}
& \left\|\mathbf{u}^{h_{l-1}}-\mathbf{u}\right\|_{a} \leq C \delta_{h_{l-1}} \\
& \left|\lambda^{h_{l-1}}-\lambda\right| \leq C \delta_{h_{l-1}}^{2}
\end{aligned}
$$

where $\mathbf{u}$ is given by (3.2) with $\mathbf{u}_{j, h_{l-1}}-\mathbf{u}_{j}^{0}$ satisfying (2.6)-(2.7). Define

$$
\overline{\mathbf{u}}=\mathbf{u} /\|\mathbf{u}\|_{a} \in \widehat{M}(\lambda) .
$$

Using (3.1) and (3.9), we deduce that $\mathbf{u}^{h_{l-1}}$ approximates $\overline{\mathbf{u}}$ and there holds

$$
\left\|\mathbf{u}^{h_{l-1}}-\overline{\mathbf{u}}\right\|_{a} \leq C \delta_{h_{l-1}} .
$$

Substituting (3.10)-(3.12) into (3.3)-(3.4), from Condition 3.1, we get

$$
\left\|\mathbf{u}^{h_{l}}-\mathbf{u}\right\|_{a} \leq C\left(\delta_{h_{l-1}}^{3}+\delta_{h_{l}}\right) \leq C \delta_{h_{l^{\prime}}}
$$

and

$$
\left|\lambda^{h_{l}}-\lambda\right| \leq C \delta_{h_{l}}^{2}
$$

The proof is complete.

\section{Adaptive finite element method}

To efficiently compute low regularity eigenpairs, we resort to the adaptive scheme $[7,8$, $12,15,20]$. The standard procedure of adaptive finite element method is to iteratively implement the following steps (see, e.g. [21])

$$
\text { Solve } \longrightarrow \text { Estimate } \longrightarrow \text { Mark } \longrightarrow \text { Refine }
$$

i.e., 1) given a mesh $\pi_{h_{l}}$, solve the problem on $\pi_{h_{l}} ; 2$ ) estimate the error of the solution by some a posteriori error estimator; 3 ) mark the subset $\widehat{\pi}_{h_{l}}$ of $\pi_{h_{l}}$ on which the error is the most needed to control using some marking strategy; and 4) refine $\widehat{\pi}_{h_{l}}$ to get the new mesh $\pi_{h_{l+1}}$. 
We derive an a posteriori error estimate following the line of [22] and then propose an a posteriori error indicator of the residual. To this end, we first prove a superconvergence result which will be used later. Define

$$
\mathbf{u}^{*}=\sum_{j=k}^{k+q-1} a\left(\mathbf{u}^{h_{l}}, \mathbf{u}_{j, h_{l}}\right) \mathbf{u}_{j, h_{l}} .
$$

Theorem 4.1. Under the conditions of Theorem 3.4, there holds

$$
\left\|\mathbf{u}^{h_{l}}-\mathbf{u}^{*}\right\|_{a} \leq C \delta_{h_{l}}^{3 / t_{l}}
$$

Proof. Step 3 of Algorithm 3.1 with $i=l$ is equivalent to: Find $\mathbf{u}^{\prime} \in U_{h_{l}}$ such that

$$
\mathbf{u}^{\prime}=T_{h_{l}}\left(\lambda^{h_{l-1}} \mathbf{u}^{\prime}+\mathbf{u}^{h_{l-1}}\right),
$$

and $\mathbf{u}^{h_{l}}=\mathbf{u}^{\prime} /\left\|\mathbf{u}^{\prime}\right\|_{a}$. That is

$$
\left(\left(\lambda^{h_{l-1}}\right)^{-1}-T_{h_{l}}\right) \mathbf{u}^{\prime}=\left(\lambda^{h_{l-1}}\right)^{-1} T_{h_{l}} \mathbf{u}^{h_{l-1}}, \quad \mathbf{u}^{h_{l}}=\mathbf{u}^{\prime} /\left\|\mathbf{u}^{\prime}\right\|_{a} .
$$

Denote

$$
\begin{aligned}
& v_{0}=\left(\lambda^{h_{l-1}}\right)^{-1}, \quad \mathbf{u}_{0}=\lambda^{h_{l-1}} T_{h_{l}} \mathbf{u}^{h_{l-1}} /\left\|\lambda^{h_{l-1}} T_{h_{l}} \mathbf{u}^{h_{l-1}}\right\|_{a}, \\
& \mathbf{u}^{\mathcal{s}}=\lambda^{h_{l-1}} \mathbf{u}^{\prime} /\left\|\lambda^{h_{l-1}} T_{h_{l}} \mathbf{u}^{h_{l-1}}\right\|_{a} .
\end{aligned}
$$

Then Step 3 of Algorithm 3.1 is equivalent to the following:

$$
\left(v_{0}-T_{h_{l}}\right) \mathbf{u}^{s}=\mathbf{u}_{0}, \quad \mathbf{u}^{h_{l}}=\mathbf{u}^{s} /\left\|\mathbf{u}^{s}\right\|_{a} .
$$

Let $\overline{\mathbf{u}}$ be the same as in (3.11). We have that

$$
\begin{aligned}
\left\|\lambda^{h_{l-1}} T_{h_{l}} \overline{\mathbf{u}}-\overline{\mathbf{u}}\right\|_{a} & =\left\|\lambda^{h_{l-1}} T_{h_{l}} \overline{\mathbf{u}}-\lambda T \overline{\mathbf{u}}\right\|_{a} \\
& \lesssim \lambda\left\|\left(T-T_{h_{l}}\right) \overline{\mathbf{u}}\right\|_{a}+\left\|\left(\lambda-\lambda^{h_{l-1}}\right) T_{h_{l}} \overline{\mathbf{u}}\right\|_{a} \\
& \lesssim \delta_{h_{l}}(\lambda)\|\overline{\mathbf{u}}\|_{a}+\left|\lambda^{h_{l-1}}-\lambda\right|\|\overline{\mathbf{u}}\|_{b} \\
& \lesssim \delta_{h_{l}}(\lambda)+\left|\lambda^{h_{l-1}}-\lambda\right| .
\end{aligned}
$$

Here $a \lesssim b$ denotes $a \leq C b$ for some constant $C$. By the boundedness of the operator $T_{h_{l}}$, we have

$$
\begin{aligned}
\left\|\mathbf{u}_{0}-\overline{\mathbf{u}}\right\|_{a} & \leq 2\left\|\lambda^{h_{l-1}} T_{h_{l}} \mathbf{u}^{h_{l-1}}-\overline{\mathbf{u}}\right\|_{a} \\
& \leq 2\left(\left\|\lambda^{h_{l-1}} T_{h_{l}} \overline{\mathbf{u}}-\overline{\mathbf{u}}\right\|_{a}+\left\|\lambda^{h_{l-1}} T_{h_{l}}\left(\overline{\mathbf{u}}-\mathbf{u}^{h_{l-1}}\right)\right\|_{a}\right) \\
& \lesssim\left\|\lambda^{h_{l-1}} T_{h_{l}} \overline{\mathbf{u}}-\overline{\mathbf{u}}\right\|_{a}+\left\|\overline{\mathbf{u}}-\mathbf{u}^{h_{l-1}}\right\|_{b} .
\end{aligned}
$$


Next we shall verify the conditions of Lemma 3.2. The estimates (4.5), (4.6) and (2.6) lead to

$$
\begin{aligned}
\operatorname{dist}\left(\mathbf{u}_{0}, M_{h_{l}}(\lambda)\right) & \leq\left\|\mathbf{u}_{0}-\overline{\mathbf{u}}\right\|_{a}+\operatorname{dist}\left(\overline{\mathbf{u}}, M_{h_{l}}(\lambda)\right) \\
& \lesssim \delta_{h_{l}}(\lambda)+\left|\lambda^{h_{l-1}}-\lambda\right|+\left\|\mathbf{u}-\mathbf{u}^{h_{l-1}}\right\|_{b} \\
& \leq 1 / 2
\end{aligned}
$$

for $H$ small enough. Noting that $\lambda_{0}$ and $\lambda_{j, h_{l}}$ are approximations of $\lambda$, it is easy to verify that, when $H$ is small enough,

$$
\left|v_{k}-v_{0}\right|=\frac{\left|\lambda^{h_{l-1}}-\lambda\right|}{\left|\lambda^{h_{l-1}} \lambda\right|} \leq \frac{d}{4}
$$

and, for $j=k-1, k, k+q, j \neq 0$,

$$
\left|v_{j}-v_{j, h_{l}}\right|=\frac{\left|\lambda_{j}-\lambda_{j, h_{l}}\right|}{\left|\lambda_{j} \lambda_{j, h_{l}}\right|} \leq \frac{d}{4}
$$

Therefore the conditions of Lemma 3.2 hold and we have that

$$
\operatorname{dist}\left(\mathbf{u}^{h_{l}}, M_{h_{l}}(\lambda)\right) \leq \frac{4}{d} \max _{k \leq j \leq k+q-1}\left|v_{j, h_{l}}-v_{0}\right| \operatorname{dist}\left(\mathbf{u}_{0}, M_{h_{l}}(\lambda)\right) .
$$

Applying (2.5), we have for $j=k, k+1, \cdots, k+q-1$

$$
\begin{aligned}
\left|v_{j, h_{l}}-v_{0}\right| & =\frac{\left|\lambda^{h_{l-1}}-\lambda_{j, h_{l}}\right|}{\left|\lambda^{h_{l-1}} \lambda_{j, h_{l}}\right|} \\
& \lesssim\left|\lambda^{h_{l-1}}-\lambda\right|+\left|\lambda-\lambda_{j, h_{l}}\right| \\
& \lesssim\left|\lambda^{h_{l-1}}-\lambda\right|+\delta_{h_{l}}^{2}(\lambda) .
\end{aligned}
$$

Substituting (4.7) and (4.9) into (4.8), we have

$$
\operatorname{dist}\left(\mathbf{u}^{h_{l}}, M_{h_{l}}(\lambda)\right) \lesssim\left(\left|\lambda^{h_{l-1}}-\lambda\right|+\delta_{h_{l}}^{2}(\lambda)\right)\left(\delta_{h_{l}}(\lambda)+\left|\lambda^{h_{l-1}}-\lambda\right|+\left\|\overline{\mathbf{u}}-\mathbf{u}^{h_{l-1}}\right\|\right) .
$$

By the definition of $\mathbf{u}^{*}$ in (4.1), we have $\left\|\mathbf{u}^{h_{l}}-\mathbf{u}^{*}\right\|_{a}=\operatorname{dist}\left(\mathbf{u}^{h_{l}}, M_{h_{l}}(\lambda)\right)$ and the assertion can be derived using Theorem 3.4 and Condition 3.1.

Theorem 4.1 indicates that $\mathbf{u}^{*}$ approximates $\mathbf{u}^{h_{l}}$. Therefore the a posteriori estimate for $\left\|\mathbf{u}^{h_{l}}-\mathbf{u}\right\|_{a}$ can be derived using that of $\left\|\mathbf{u}^{*}-\mathbf{u}\right\|_{a}$. Define the residuals:

$$
\begin{aligned}
& \widehat{R}_{\mathcal{K}}\left(\mathbf{u}^{*}\right)=\nabla \cdot \sigma\left(\mathbf{u}^{*}\right)+\sum_{j=k}^{k+q-1} a\left(\mathbf{u}^{h_{l}}, \mathbf{u}_{j, h_{l}}\right) \lambda_{j, h_{l}} \rho \mathbf{u}_{j, h_{l},} \\
& \widehat{R}_{\partial \kappa}\left(\mathbf{u}^{*}\right)= \begin{cases}J_{F}\left(\mathbf{n}_{F} \cdot \sigma\left(\mathbf{u}^{*}\right)\right), & F \subset \partial \kappa \cap \mathcal{E}_{\Omega}, \\
0, & F \subset \partial \kappa \cap \mathcal{E}_{D},\end{cases}
\end{aligned}
$$


where, for an edge $F$ of the element $\kappa, \mathbf{n}_{F}$ denotes the unit outward normal of $F, J_{F}(\phi)$ denotes the jump of a given function $\phi$ across $F$ along $\mathbf{n}_{F}, \mathcal{E}_{\Omega}$ and $\mathcal{E}_{D}$ are the sets of interior edges of $\Omega$ and boundary edges on $\partial \Omega$, respectively. For $\kappa \in \pi_{h_{l}}$, define the local error indicator

$$
\widehat{\eta}_{h_{l}}\left(\mathbf{u}^{*}, \kappa\right)=\left\{d_{\kappa, \min }^{-1} h_{\kappa}^{2}\left\|\widehat{R}_{\kappa}\left(\mathbf{u}^{*}\right)\right\|_{0, \kappa}^{2}+d_{\kappa, \min }^{-1} h_{\kappa}\left\|\widehat{R}_{\partial \kappa}\left(\mathbf{u}^{*}\right)\right\|_{0, \partial \kappa}^{2}\right\}^{1 / 2},
$$

where $d_{\kappa, \min }$ is the minimum eigenvalue of the matrix

$$
D:=\left[\begin{array}{ccc}
\bar{\lambda}+2 \bar{\mu} & \bar{\lambda} & 0 \\
\bar{\lambda} & \bar{\lambda}+2 \bar{\mu} & 0 \\
0 & 0 & \bar{\mu}
\end{array}\right]
$$

on the element $\kappa$.

The global error indicator is then given by

$$
\widehat{\eta}_{h_{l}}\left(\mathbf{u}^{*}, \Omega\right)=\left\{\sum_{\kappa \in \pi_{h_{l}}} \widehat{\eta}_{h_{l}}^{2}\left(\mathbf{u}^{*}, \kappa\right)\right\}^{1 / 2} .
$$

Denote

$$
e(\mathbf{u})=\mathbf{u}-\mathbf{u}^{*}, \quad e\left(\mathbf{u}_{j}^{0}\right)=\mathbf{u}_{j}^{0}-\mathbf{u}_{j, h_{l}},
$$

where $\mathbf{u}_{j}^{0}$ is defined as in (3.2).

Lemma 4.1. Let $\mathbf{u}$ and $e(\mathbf{u})$ be defined as in (3.2) and (4.13), respectively. Then there hold

$$
\begin{aligned}
& \|e(\mathbf{u})\|_{a} \leq C\left(\widehat{\eta}_{h_{l}}\left(\mathbf{u}^{*}, \Omega\right)+\sum_{j=k}^{k+q-1}\left\|\lambda \mathbf{u}_{j}^{0}-\lambda_{j, h_{l}} \mathbf{u}_{j, h_{l}}\right\|_{b}\right), \\
& \widehat{\eta}_{h_{l}}\left(\mathbf{u}^{*}, \Omega\right) \leq C\left(\|e(\mathbf{u})\|_{a}+h_{l} \sum_{j=k}^{k+q-1}\left\|\lambda \mathbf{u}_{j}^{0}-\lambda_{j, h_{l}} \mathbf{u}_{j, h_{l}}\right\|_{b}\right) .
\end{aligned}
$$

Proof. Choose $\mathbf{v} \in U_{h_{l}}$ as a test function. Subtraction of (2.3) from (2.2) gives

$$
a\left(e\left(\mathbf{u}_{j}^{0}\right), \mathbf{v}\right)=b\left(\lambda \mathbf{u}_{j}^{0}-\lambda_{j, h_{l}} \mathbf{u}_{j, h_{l}}, \mathbf{v}\right), \quad \forall \mathbf{v} \in U_{h_{l}},
$$

from which and (3.2) it follows

$$
a(e(\mathbf{u}), \mathbf{v})=b\left(\lambda \mathbf{u}-\sum_{j=k}^{k+q-1} a\left(\mathbf{u}^{h_{l}}, \mathbf{u}_{j, h_{l}}\right) \lambda_{j, h_{l}} \mathbf{u}_{j, h_{l}}, \mathbf{v}\right), \quad \forall \mathbf{v} \in U_{h_{l}} .
$$


Let $\mathbf{w}=e(\mathbf{u}) /\|e(\mathbf{u})\|_{a}$, then $\|e(\mathbf{u})\|_{a}=a(e(\mathbf{u}), \mathbf{w})$. Let $\mathbf{w}^{I} \in U_{h_{l}}$ be the Clément interpolation of $\mathbf{w}$ (see, e.g. $[3,6])$. We deduce from the above equality that

$$
\begin{aligned}
\|e(\mathbf{u})\|_{a} & =a\left(e(\mathbf{u}), \mathbf{w}-\mathbf{w}^{I}\right)+b\left(\lambda \mathbf{u}-\sum_{j=k}^{k+q-1} a\left(\mathbf{u}^{h_{l}}, \mathbf{u}_{j, h_{l}}\right) \lambda_{j, h_{l}} \mathbf{u}_{j, h_{l}}, \mathbf{w}^{I}\right) \\
& =a\left(e(\mathbf{u}), \mathbf{w}-\mathbf{w}^{I}\right)-b\left(\lambda \mathbf{u}-\sum_{j=k}^{k+q-1} a\left(\mathbf{u}^{h_{l}}, \mathbf{u}_{j, h_{l}}\right) \lambda_{j, h_{l}} \mathbf{u}_{j, h_{l}}, \mathbf{w}-\mathbf{w}^{I}-\mathbf{w}\right) .
\end{aligned}
$$

Define the auxiliary residuals associated with the element $\kappa$ :

$$
\begin{aligned}
& \widehat{R}_{\mathcal{K}}\left(\mathbf{u}_{j, h_{l}}\right)=\nabla \cdot \sigma\left(\mathbf{u}_{j, h_{l}}\right)+\lambda_{j, h_{l}} \rho \mathbf{u}_{j, h_{l},} \\
& \widehat{R}_{\partial \kappa}\left(\mathbf{u}_{j, h_{l}}\right)= \begin{cases}J_{F}\left(\mathbf{n}_{F} \cdot \sigma\left(\mathbf{u}_{j, h_{l}}\right)\right), & F \subset \partial \kappa \cap \mathcal{E}_{\Omega}, \\
0, & F \subset \partial \kappa \cap \mathcal{E}_{D} .\end{cases}
\end{aligned}
$$

Integrating by parts (see Lemma 4 in [22]), we get

$$
\begin{aligned}
& a\left(e\left(\mathbf{u}_{j}^{0}\right), \mathbf{w}-\mathbf{w}^{I}\right)-b\left(\lambda \mathbf{u}_{j}^{0}-\lambda_{j, h_{l}} \mathbf{u}_{j, h_{l}}, \mathbf{w}-\mathbf{w}^{I}\right) \\
= & \sum_{\kappa \in \pi_{h_{l}}}\left\{\left(\widehat{R}_{\kappa}\left(\mathbf{u}_{j, h_{l}}\right), \mathbf{w}-\mathbf{w}^{I}\right)_{\kappa}-\frac{1}{2}\left\langle\widehat{R}_{\partial \kappa}\left(\mathbf{u}_{j, h_{l}}\right), \mathbf{w}-\mathbf{w}^{I}\right\rangle_{\partial \kappa}\right\}
\end{aligned}
$$

for $j=k, k+1, \cdots, k+q-1$, where $\langle\cdot, \cdot\rangle_{\partial \kappa}$ denotes the $L^{2}$ inner product on $\partial \kappa$. Hence

$$
\begin{aligned}
& a\left(e(\mathbf{u}), \mathbf{w}-\mathbf{w}^{I}\right)-b\left(\lambda \mathbf{u}-\sum_{j=k}^{k+q-1} a\left(\mathbf{u}^{h_{l}}, \mathbf{u}_{j, h_{l}}\right) \lambda_{j, h_{l}} \mathbf{u}_{j, h_{l}}, \mathbf{w}-\mathbf{w}^{I}\right) \\
= & \sum_{\kappa \in \pi_{h_{l}}}\left\{\left(\widehat{R}_{\mathcal{K}}\left(\mathbf{u}^{*}\right), \mathbf{w}-\mathbf{w}^{I}\right)_{\kappa}-\frac{1}{2}\left\langle\widehat{R}_{\partial \kappa}\left(\mathbf{u}^{*}\right), \mathbf{w}-\mathbf{w}^{I}\right\rangle_{\partial \kappa}\right\} .
\end{aligned}
$$

From Cauchy-Schwarz inequality, we obtain

$$
\begin{gathered}
a\left(e(\mathbf{u}), \mathbf{w}-\mathbf{w}^{I}\right)-b\left(\lambda \mathbf{u}-\sum_{j=k}^{k+q-1} a\left(\mathbf{u}^{h_{l}}, \mathbf{u}_{j, h_{l}}\right) \lambda_{j, h_{l}} \mathbf{u}_{j, h_{l}}, \mathbf{w}-\mathbf{w}^{I}\right) \\
\leq C\left\{\sum_{\kappa \in \pi_{h_{l}}}\left(d_{\kappa, \min } h_{\mathcal{\kappa}}^{-2}\left\|\mathbf{w}-\mathbf{w}^{I}\right\|_{0, \kappa}^{2}+d_{\kappa, \min } h_{\mathcal{\kappa}}^{-1}\left\|\mathbf{w}-\mathbf{w}^{I}\right\|_{0, \partial \kappa}^{2}\right)\right\}^{1 / 2} \\
\cdot\left\{\sum_{\kappa \in \pi_{h_{l}}}\left(d_{\kappa, \min }^{-1} h_{\kappa}^{2}\left\|\widehat{R}_{\kappa}\left(\mathbf{u}^{*}\right)\right\|_{0, \kappa}^{2}+d_{\kappa, \min }^{-1} h_{\kappa}\left\|\widehat{R}_{\partial \kappa}\left(\mathbf{u}^{*}\right)\right\|_{\partial \kappa}^{2}\right)\right\}^{1 / 2} .
\end{gathered}
$$

Using the Clément interpolation estimate we have

$$
d_{\kappa, \min } h_{\kappa}^{-2}\left\|\mathbf{w}-\mathbf{w}^{I}\right\|_{0, \kappa}^{2}+d_{\kappa, \min } h_{\kappa}^{-1}\left\|\mathbf{w}-\mathbf{w}^{I}\right\|_{0, \partial \kappa}^{2} \leq C\left(d_{\kappa, \min }\|\mathbf{w}\|_{0, \kappa}^{2}+d_{\kappa, \min }|\mathbf{w}|_{1, \kappa}^{2}\right) .
$$


For $\widetilde{\varepsilon}(\mathbf{w})=\left(\partial_{x} w_{1}, \partial_{y} w_{2}, \partial_{x} w_{2}+\partial_{y} w_{1}\right)^{\top}$, note that there hold

$$
d_{\min }\|\widetilde{\varepsilon}(\mathbf{w})\|_{0}^{2} \leq \int_{\Omega} D \widetilde{\varepsilon}(\mathbf{w}) \cdot \widetilde{\varepsilon}(\mathbf{w})
$$

and (see for example [3])

$$
\|\mathbf{w}\|_{1}^{2} \leq C\|\widetilde{\varepsilon}(\mathbf{w})\|_{0}^{2} .
$$

Summing over all elements we get

$$
\begin{aligned}
& \sum_{\kappa \in \pi_{h_{l}}} d_{\kappa, \min } h_{\kappa}^{-2}\left\|\mathbf{w}-\mathbf{w}^{I}\right\|_{0, \kappa}^{2}+d_{\kappa, \min } h_{\kappa}^{-1}\left\|\mathbf{w}-\mathbf{w}^{I}\right\|_{0, \partial \kappa}^{2} \\
\leq & C d_{\max }\|\mathbf{w}\|_{1}^{2} \leq C d_{\max } / d_{\min },
\end{aligned}
$$

where $d_{\max }=\max _{\kappa} d_{\kappa, \max }$ and $d_{\min }=\min _{\kappa} d_{\kappa, \min }$ with $d_{\kappa, \max }$ being the maximum eigenvalue of $D$ on the element $\kappa$. Using this inequality and (4.17)-(4.18), we have

$$
a\left(e(\mathbf{u}), \mathbf{w}-\mathbf{w}^{I}\right)-b\left(\lambda \mathbf{u}-\sum_{j=k}^{k+q-1} a\left(\mathbf{u}^{h_{l}}, \mathbf{u}_{j, h_{l}}\right) \lambda_{j, h_{l}} \mathbf{u}_{j, h_{l}}, \mathbf{w}-\mathbf{w}^{I}\right) \leq C \widehat{\eta}_{h_{l}}\left(\mathbf{u}^{*}, \Omega\right) .
$$

Using (4.16) and (4.19), we have

$$
\|e(\mathbf{u})\|_{a} \leq C\left(\widehat{\eta}_{h_{l}}\left(\mathbf{u}^{*}, \Omega\right)+\left\|\sum_{j=k}^{k+q-1} a\left(\mathbf{u}^{h_{l}}, \mathbf{u}_{j, h_{l}}\right)\left(\lambda \mathbf{u}_{j}^{0}-\lambda_{j, h_{l}} \mathbf{u}_{j, h_{l}}\right)\right\|_{b}\right) .
$$

This yields (4.14). As for the reliability of $\widehat{\eta}_{h_{l}}\left(\mathbf{u}^{*}, \Omega\right)$, referring (20) in [22], we can obtain that

$$
\begin{aligned}
& \sqrt{d_{\kappa, \min }^{-1}} h_{\kappa}\left\|\widehat{R}_{\kappa}\left(\mathbf{u}^{*}\right)\right\|_{0, \kappa}+\sqrt{d_{\kappa, \min }^{-1}} \sqrt{h_{\kappa}}\left\|\widehat{R}_{\partial \kappa}\left(\mathbf{u}^{*}\right)\right\|_{0, \partial \kappa} \\
& \leq C\left(\sqrt{\frac{d_{\kappa, \max }}{d_{\kappa, \text { min }}}}\|e(\mathbf{u})\|_{a, \omega_{\kappa}}+\frac{h_{\omega_{\kappa}}}{\sqrt{d_{\kappa, \min }}}\left\|\sum_{j=k}^{k+q-1} a\left(\mathbf{u}^{h_{l}}, \mathbf{u}_{j, h_{l}}\right)\left(\lambda \mathbf{u}_{j}^{0}-\lambda_{j, h_{l}} \mathbf{u}_{j, h_{l}}\right)\right\|_{b, \omega_{\kappa}}\right),
\end{aligned}
$$

where $\omega_{\kappa}$ is the union of the elements sharing at least one face with $\kappa$. Summing over all elements, we get

$$
\widehat{\eta}_{h_{l}}\left(\mathbf{u}^{*}, \Omega\right) \leq C \sqrt{\frac{d_{\max }}{d_{\min }}}\|e(\mathbf{u})\|_{a}+h_{l} \sqrt{d_{\min }^{-1}} \sum_{j=k}^{k+q-1}\left\|\lambda \mathbf{u}_{j}^{0}-\lambda_{j, h_{l}} \mathbf{u}_{j, h_{l}}\right\|_{b} .
$$

The proof is complete. 
Now we are in a position to discuss the a posteriori estimate of $\left\|\mathbf{u}^{h_{l}}-\mathbf{u}\right\|_{a}$. We define the local error indicator for $\mathbf{u}^{h_{l}}$ as follows: For $\kappa \in \pi_{h_{l}}$,

$$
\begin{aligned}
& R_{\mathcal{K}}\left(\mathbf{u}^{h_{l}}\right)=\nabla \cdot \sigma\left(\mathbf{u}^{h_{l}}\right)+\lambda^{h_{l}} \rho \mathbf{u}^{h_{l},} \\
& R_{\partial \kappa}\left(\mathbf{u}^{h_{l}}\right)= \begin{cases}J_{F}\left(\mathbf{n}_{F} \cdot \sigma\left(\mathbf{u}^{h_{l}}\right)\right), & F \subset \partial \kappa \cap \mathcal{E}_{\Omega}, \\
0, & F \subset \partial \kappa \cap \mathcal{E}_{D},\end{cases} \\
& \eta_{h_{l}}\left(\mathbf{u}^{\left.h_{l}, \kappa\right)}=\left\{d_{\kappa, \text { min }}^{-1} h_{\kappa}^{2}\left\|R_{\mathcal{K}}\left(\mathbf{u}^{h_{l}}\right)\right\|_{0, \kappa}^{2}+d_{\kappa, \min }^{-1} h_{\mathcal{\kappa}}\left\|R_{\partial \kappa}\left(\mathbf{u}^{h_{l}}\right)\right\|_{0, \partial \kappa}^{2}\right\}^{1 / 2} .\right.
\end{aligned}
$$

Then the global error indicator is given by

$$
\eta_{h_{l}}\left(\mathbf{u}^{h_{l}}, \Omega\right)=\left\{\sum_{\kappa \in \pi_{h_{l}}} \eta_{h_{l}}^{2}\left(\mathbf{u}^{\left.h_{l}, \kappa\right)}\right\}^{1 / 2} .\right.
$$

Theorem 4.2. Let $\mathbf{u}$ be defined by (3.2). Under the conditions of Theorem 3.4, there hold

$$
\begin{aligned}
& \left\|\mathbf{u}-\mathbf{u}^{h_{l}}\right\|_{a} \leq C\left(\eta_{h_{l}}\left(\mathbf{u}^{h_{l}}, \Omega\right)+\delta_{h_{l}}^{3 / t_{l}}+h_{l}^{r} \delta_{h_{l}}\right), \\
& C \eta_{h_{l}}\left(\mathbf{u}^{h_{l}}, \Omega\right) \leq\left\|\mathbf{u}-\mathbf{u}^{h_{l}}\right\|_{a}+\delta_{h_{l}}^{3 / t_{l}}+h_{l}^{r+1} \delta_{h_{h^{\prime}}} \\
& \left|\lambda^{h_{l}}-\lambda\right| \leq C\left(\eta_{h_{l}}^{2}\left(\mathbf{u}^{h_{l}}, \Omega\right)+\delta_{h_{l}}^{6 / t_{l}}+h_{l}^{2 r} \delta_{h_{l}}^{2}\right) .
\end{aligned}
$$

Proof. By the triangle inequality,

$$
\left\|\mathbf{u}-\mathbf{u}^{h_{l}}\right\|_{a} \leq\left\|\mathbf{u}-\mathbf{u}^{*}\right\|_{a}+\left\|\mathbf{u}^{*}-\mathbf{u}^{h_{l}}\right\|_{a} .
$$

Using (4.14), from (4.24), we get

$$
\begin{aligned}
\left\|\mathbf{u}-\mathbf{u}^{h_{l}}\right\|_{a} & \leq C\left(\widehat{\eta}_{h_{l}}\left(\mathbf{u}^{*}, \Omega\right)+\sum_{j=k}^{k+q-1}\left\|\lambda \mathbf{u}_{j}^{0}-\lambda_{j, h_{l}} \mathbf{u}_{j, h_{l}}\right\|_{b}\right)+\left\|\mathbf{u}^{*}-\mathbf{u}^{h_{l}}\right\|_{a} \\
& =C\left(\eta_{h_{l}}\left(\mathbf{u}^{h_{l}}, \Omega\right)+\sum_{j=k}^{k+q-1}\left\|\lambda \mathbf{u}_{j}^{0}-\lambda_{j, h_{l}} \mathbf{u}_{j, h_{l}}\right\|_{b}\right)+R_{1}+\left\|\mathbf{u}^{*}-\mathbf{u}^{h_{l}}\right\|_{a},
\end{aligned}
$$

where $R_{1}=C\left(\widehat{\eta}_{h_{l}}\left(\mathbf{u}^{*}, \Omega\right)-\eta_{h_{l}}\left(\mathbf{u}^{h_{l}}, \Omega\right)\right)$. By the triangle inequality,

$$
\left|R_{1}\right| \leq C\left(\sum_{\kappa \in \pi_{h_{l}}}\left(\widehat{\eta}_{h_{l}}\left(\mathbf{u}^{*}, \kappa\right)-\eta_{h_{l}}\left(\mathbf{u}^{\left.h_{l}, \kappa\right)}\right)^{2}\right)^{1 / 2}\right.
$$


Using again the triangle inequality, we obtain that

$$
\begin{aligned}
& \left|\widehat{\eta}_{h_{l}}\left(\mathbf{u}^{*}, \kappa\right)-\eta_{h_{l}}\left(\mathbf{u}^{h_{l}}, \kappa\right)\right| \\
\leq & C\left\{h_{\kappa}^{2}\left\|\widehat{R}_{\kappa}\left(\mathbf{u}^{*}\right)-R_{\kappa}\left(\mathbf{u}^{h_{l}}\right)\right\|_{0, \kappa}^{2}+h_{\kappa}\left\|\widehat{R}_{\partial \kappa}\left(\mathbf{u}^{*}\right)-R_{\partial \kappa}\left(\mathbf{u}^{h_{l}}\right)\right\|_{0, \partial \kappa}^{2}\right\}^{1 / 2} \\
\leq & \left\{h_{\kappa}^{2}\left\|\nabla \cdot \sigma\left(\mathbf{u}^{*}-\mathbf{u}^{h_{l}}\right)\right\|_{0, \kappa}^{2}+h_{\kappa}^{2}\left\|\lambda^{h_{l}} \mathbf{u}^{h_{l}}-\sum_{j=k}^{k+q-1} a\left(\mathbf{u}^{h_{l}}, \mathbf{u}_{j, h_{l}}\right) \lambda_{j, h_{l}} \mathbf{u}_{j, h_{l}}\right\|_{0, \kappa}^{2}\right. \\
& \left.+h_{\kappa} \sum_{F \subset \partial \kappa}\left\|J_{F}\left(\mathbf{n}_{F} \cdot \sigma\left(\mathbf{u}^{*}-\mathbf{u}^{h_{l}}\right)\right)\right\|_{0, \partial \kappa}^{2}\right\}^{1 / 2},
\end{aligned}
$$

which together with the inverse estimates and trace theorem yields

$$
\begin{aligned}
& \left|\widehat{\eta}_{h_{l}}\left(\mathbf{u}^{*}, \kappa\right)-\eta_{h_{l}}\left(\mathbf{u}^{h_{l}}, \kappa\right)\right| \\
\leq & C\left(\left|\mathbf{u}^{*}-\mathbf{u}^{h_{l}}\right|_{1, \kappa}+h_{\kappa}|| \lambda^{h_{l}} \mathbf{u}^{h_{l}}-\sum_{j=k}^{k+q-1} a\left(u^{h_{l}}, \mathbf{u}_{j, h_{l}}\right) \lambda_{j, h_{l}} \mathbf{u}_{j, h_{l}} \|_{0, \kappa}\right) .
\end{aligned}
$$

Since we have

$$
\begin{aligned}
& \left\|\lambda^{h_{l}} \mathbf{u}^{h_{l}}-\sum_{j=k}^{k+q-1} a\left(\mathbf{u}^{h_{l}}, \mathbf{u}_{j, h_{l}}\right) \lambda_{j, h_{l}} \mathbf{u}_{j, h_{l}}\right\|_{0, \kappa} \\
\leq & \left\|\lambda^{h_{l}}\left(\mathbf{u}^{h_{l}}-\mathbf{u}^{*}\right)\right\|_{0, \kappa}+\left\|\sum_{j=k}^{k+q-1} a\left(\mathbf{u}^{h_{l}}, \mathbf{u}_{j, h_{l}}\right)\left(\lambda_{j, h_{l}}-\lambda^{h_{l}}\right) \mathbf{u}_{j, h_{l}}\right\|_{0, \kappa} \\
\leq C & \left(\left\|\mathbf{u}^{h_{l}}-\mathbf{u}^{*}\right\|_{0, \kappa}+\sum_{j=k}^{k+q-1}\left|\lambda_{j, h_{l}}-\lambda^{h_{l}}\right|\left\|\mathbf{u}_{j, h_{l}}\right\|_{0, \kappa}\right),
\end{aligned}
$$

(2.5) and (3.6) lead to

$$
\left|\widehat{\eta}_{h_{l}}\left(\mathbf{u}^{*}, \kappa\right)-\eta_{h_{l}}\left(\mathbf{u}^{h_{l}}, \kappa\right)\right| \leq C\left(\left\|\mathbf{u}^{*}-\mathbf{u}^{h_{l}}\right\|_{1, \kappa}+\sum_{j=k}^{k+q-1} h_{\kappa} \delta_{h_{l}}^{2}\left\|\mathbf{u}_{j, h_{l}}\right\|_{0, \kappa}\right) .
$$

Substitution of the above formula into (4.26), from (2.5), (2.6) and (4.2) we obtain

$$
\left|R_{1}\right| \leq C\left(\left\|\mathbf{u}^{*}-\mathbf{u}^{h_{l}}\right\|_{a}+\sum_{j=k}^{k+q-1} h_{l} \delta_{h_{l}}^{2}\left\|\mathbf{u}_{j, h_{l}}\right\|_{b}\right) \leq C \max \left(\delta_{h_{l}}^{3 / t_{l}}, h_{l} \delta_{h_{l}}^{2}\right)
$$

and

$$
\left\|\mathbf{u}^{*}-\mathbf{u}^{h_{l}}\right\|_{a} \leq C \delta_{h_{l}}^{3 / t_{l}}, \quad \sum_{j=k}^{k+q-1}\left\|\lambda \mathbf{u}_{j}^{0}-\lambda_{j, h_{l}} \mathbf{u}_{j, h_{l}}\right\|_{b} \leq C h_{l}^{r} \delta_{h_{l}} .
$$


Substituting the above two formulae into (4.25) yields (4.21). The combination of (3.4) and (4.21) yields (4.22). From (4.15) we have

$$
\begin{gathered}
C \eta_{h_{l}}\left(\mathbf{u}^{\left.h_{l}, \Omega\right) \leq} \leq\left\|\mathbf{u}-\mathbf{u}^{h_{l}}\right\|_{a}-C\left(\widehat{\eta}_{h_{l}}\left(\mathbf{u}^{*}, \Omega\right)-\eta_{h_{l}}\left(\mathbf{u}^{h_{l}}, \Omega\right)\right)+\left\|\mathbf{u}^{*}-\mathbf{u}^{h_{l}}\right\|_{a}\right. \\
+\sum_{j=k}^{k+q-1} h_{l}\left\|\lambda \mathbf{u}_{j}^{0}-\lambda_{j, h_{l}} \mathbf{u}_{j, h_{l}}\right\|_{b} .
\end{gathered}
$$

The assertion (4.23) can be derived using the estimate of $\left|R_{1}\right|$ and (4.28).

Using the a posteriori error estimator defined above, i.e.,

$$
\eta_{h_{l}}\left(\mathbf{u}^{h_{l}}, \kappa\right)=\left\{d_{\kappa, \min }^{-1} h_{\mathcal{\kappa}}^{2}\left\|R_{\kappa}\left(\mathbf{u}^{h_{l}}\right)\right\|_{0, \kappa}^{2}+d_{\kappa, \min }^{-1} h_{\mathcal{\kappa}}\left\|R_{\partial \kappa}\left(\mathbf{u}^{h_{l}}\right)\right\|_{0, \partial \kappa}^{2}\right\}^{1 / 2},
$$

the marking strategy can be defined as

$$
\sum_{\kappa \in \widehat{\pi}_{h_{l}}} \eta_{h_{l}}\left(\mathbf{u}^{h_{l}}, \kappa\right) \geqslant \theta \sum_{\kappa \in \pi_{h_{l}}} \eta_{h_{l}}\left(\mathbf{u}^{h_{l}}, \kappa\right), \quad \text { for some } \quad 0<\theta<1 .
$$

The following is the adaptive algorithm.

Algorithm 4.1. Adaptive Algorithm.

1. Choose the parameter $0<\theta<1$ and the tolerance $\varepsilon$.

2. Solve (2.1) on $\pi_{h_{0}}$ to get the discrete solution $\left(\lambda^{h_{0}}, \mathbf{u}^{h_{0}}\right)$.

3. Compute the error estimator $\eta_{h_{l}}\left(\mathbf{u}^{h_{0}}, \kappa\right)$ for $\kappa \in \pi_{h_{0}}$. Let $l \leftarrow 0$.

4. While $\eta_{h_{l}}\left(\mathbf{u}^{h_{l}}, \pi_{h_{l}}\right)^{2}>\varepsilon$

a. Mark the subset $\widehat{\pi}_{h_{l}} \subset \pi_{h_{l}}$ by the marking strategy

$$
\sum_{\kappa \in \widehat{\pi}_{h_{l}}} \eta_{h_{l}}\left(\mathbf{u}^{h_{l}}, \kappa\right) \geqslant \theta \sum_{\kappa \in \pi_{h_{l}}} \eta_{h_{l}}\left(\mathbf{u}^{h_{l}}, \kappa\right)
$$

b. Refine $\widehat{\pi}_{h_{l}}$ to get the new mesh $\pi_{h_{l+1}}$.

c. Solve (2.1) on $\pi_{h_{l+1}}$ to get the discrete solution $\left(\lambda^{h_{l+1}}, \mathbf{u}^{h_{l+1}}\right)$.

d. Compute the error estimator $\eta_{h_{l}}\left(\mathbf{u}^{h_{l+1}}, \kappa\right)$ for $\kappa \in \pi_{h_{l+1}}$.

e. $l \leftarrow l+1$.

Combining the multigrid discretization Algorithm 3.1 with Algorithm 4.1, we end up with the adaptive multigrid scheme as follows.

Algorithm 4.2. Adaptive Multigrid Scheme.

1. Choose the parameter $0<\theta<1$ and the integers $M$ and $l_{0}$. 
2. Solve (2.1) on $\pi_{h_{0}}$ to get the discrete solution $\left(\lambda^{h_{0}}, \mathbf{u}^{h_{0}}\right)$. Set $l \leftarrow 0$.

3. For $l=1, \cdots, M$

a. Compute the error estimator $\eta_{h_{l}}\left(\mathbf{u}^{h_{l}}, \kappa\right)$ for $\kappa \in \pi_{h_{l}}$.

b. Mark the subset $\widehat{\pi}_{h_{l}} \subset \pi_{h_{l}}$ by the marking strategy

$$
\sum_{\kappa \in \hat{\pi}_{h_{l}}} \eta_{h_{l}}\left(\mathbf{u}^{h_{l}}, \kappa\right) \geqslant \theta \sum_{\kappa \in \pi_{h_{l}}} \eta_{h_{l}}\left(\mathbf{u}^{\left.h_{l}, \kappa\right)}\right.
$$

c. Refine $\widehat{\pi}_{h_{l}}$ to get the new mesh $\pi_{h_{l+1}}$.

d. Solve $\mathbf{u}^{h_{l+1}} \in U_{h_{l+1}}$ such that $\left\|\mathbf{u}^{h_{l+1}}\right\|_{a}=1$ and

$$
a\left(\mathbf{u}^{h_{l+1}}, \mathbf{v}\right)-\lambda^{h_{l}} b\left(\mathbf{u}^{h_{l+1}}, \mathbf{v}\right)=b\left(\mathbf{u}^{h_{l}}, \mathbf{v}\right), \quad \forall \mathbf{v} \in U_{h_{l+1}} .
$$

e. Compute $\lambda^{h_{l+1}}$ by the Rayleigh quotient

$$
\lambda^{h_{l+1}}=\frac{a\left(\mathbf{u}^{h_{l+1}}, \mathbf{u}^{h_{l+1}}\right)}{b\left(\mathbf{u}^{\left.h_{l+1}, \mathbf{u}^{h_{l+1}}\right)}\right.} .
$$

\section{$5 \quad$ Numerical experiments}

We present some numerical results for two elastic eigenvalue problems: (1) on the Lshaped domain, and (2) with discontinuous Lamé parameters. For each problem, we first compare the adaptive scheme Algorithm 4.1 and the classical finite element method on uniformly refined meshes, which shows that the adaptive algorithm is more efficient for low regularity eigenfunctions. Then we compare Algorithm 4.2 and Algorithm 4.1.

Example 5.1. Consider the elastic eigenvalue problem (2.1) on the L-shaped domain $\Omega=$ $[0,1] \times[0,1] \backslash[1 / 2,1] \times[1 / 2,1]$ with density $\rho \equiv 1$ and Lamé constants $\bar{\mu}=\bar{\lambda} \equiv 1$. We solve this problem by Algorithm 4.1 and compare with the results on uniformly refined meshes. The size of the initial mesh is $h \approx 0.1$. With the tolerance $\varepsilon=0.001$, Algorithm 4.1 ends up with 18 iterations of refinement. For comparison, we refine the initial mesh uniformly 5 times.

In order to show the convergence order, we assume that the rate of convergence (with respect to the degree of freedom) among the last two refinements, namely, $l=17$ and $l=18$ for Algorithm 4.1 and among $l=4$ and $l=5$ for the uniform refinements, has reached the optimal. That is, we assume the errors of the last two iterations of adaptive refinement decay with rate 1 , and those of uniform refinements decay with order $2 / 3$. Under this assumption, we compute the limit of the eigenvalue as $l$ goes to infinity using the computed eigenvalues and the degrees of freedom of the last two refinements. More precisely, denote by $\lambda_{l}$ the computed eigenvalue, by $N_{l}$ the degrees of freedom, by $r$ the optimal decay 


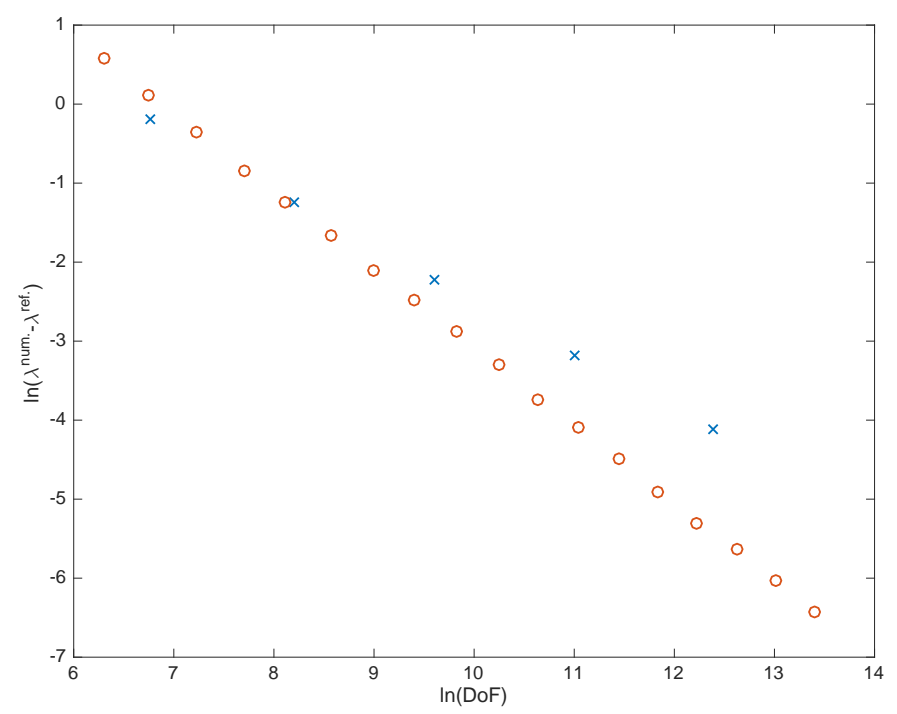

Figure 1: The difference between the first numerical eigenvalue and the reference value on the L-shaped region computed by Algorithm 4.1 and uniformly refined meshes. The circles and crosses stand for the results by using the adaptive scheme and uniformly refined meshes, respectively.

order, and by $\lambda_{*}$ the limit, and assume that the errors at $(l-1)$-th and $l$-th refinements have exactly the same rate of decay. Set

$$
\frac{\lambda_{l-1}-\lambda_{*}}{\lambda_{l}-\lambda_{*}}=\frac{N_{l-1}^{-r}}{N_{l}^{-r}}
$$

which gives us the reference value $\lambda_{*}$

$$
\lambda_{*}=\lambda_{l}+\left(\frac{N_{l-1}^{-r}}{N_{l}^{-r}}-1\right)^{-1}\left(\lambda_{l}-\lambda_{l-1}\right) .
$$

We show in Table 1 the smallest eigenvalue, degrees of freedom and convergence orders of Algorithm 4.1 and of uniformly refined meshes. It is clear that the rate of convergence with respect to the degrees of freedom is 1 for the adaptive algorithm and $2 / 3$ for the uniform refinements. Note that these rates correspond to the $\mathcal{O}\left(h^{2}\right)$ and $\mathcal{O}\left(h^{4 / 3}\right)$, respectively. In Fig. 1 we show the log-log plot of the errors against the degrees of freedom. It can be seen that the adaptive algorithm produces smaller error after the 5th iteration. The result indicates that the adaptive refinement uses much less degrees of freedom when similar accuracy is achieved. In Fig. 2 we show the initial mesh and the adaptive mesh at $l=7$. We see that the mesh is denser near the corner at $(1 / 2,1 / 2)$, where the regularity of the first eigenfunction is low. 
Table 1: The first elastic eigenvalue on the L-shaped domain using Algorithm 4.1 and uniformly refined meshes.

\begin{tabular}{||ccrc||rrrr||}
\hline$l$ & adaptive & DoF & order & $l$ & uniform & DoF & order \\
\hline 2 & 55.4925 & 858 & 1.0177 & 1 & 55.1915 & 866 & - \\
\hline 5 & 54.6566 & 3360 & 0.9233 & 2 & 54.6574 & 3618 & 0.7323 \\
\hline 8 & 54.4506 & 12032 & 0.9189 & 3 & 54.4759 & 14786 & 0.7029 \\
\hline 12 & 54.3845 & 62490 & 0.8526 & 4 & 54.4103 & 59778 & 0.6772 \\
\hline 15 & 54.3726 & 203194 & 1.0182 & 5 & 54.3851 & 240386 & 0.6667 \\
\hline ref. & 54.3676 & & & ref. & 54.3686 & & \\
\hline
\end{tabular}
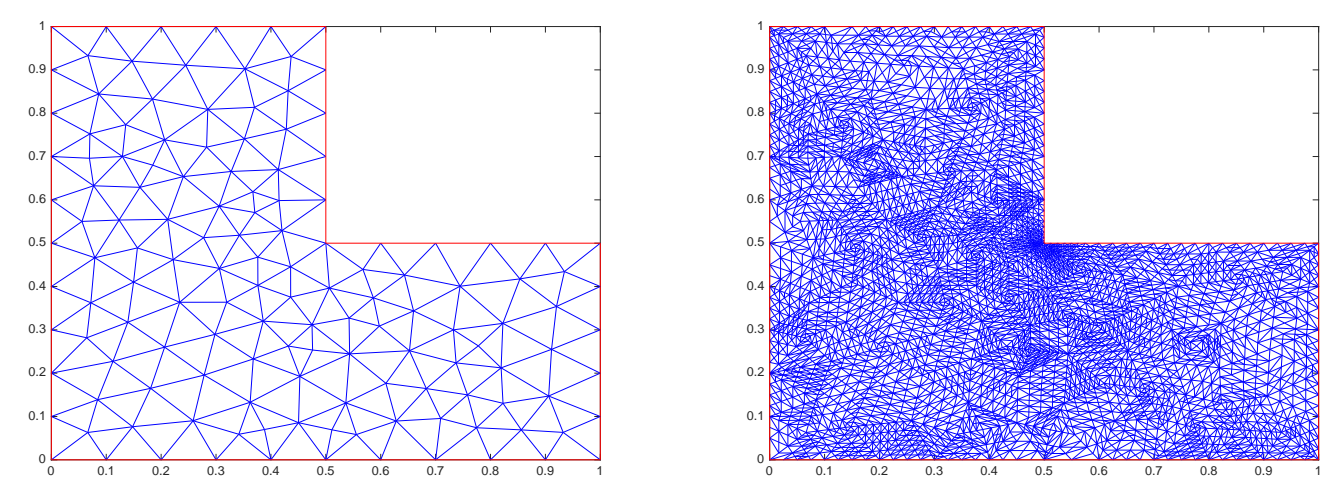

Figure 2: The meshes of Example 5.1, where the left is the initial mesh, and the right is the adaptive mesh at $l=7$.

Example 5.2. Using the same parameters as Example 5.1, we solve the eigenvalue problem using Algorithm 4.1 and Algorithm 4.2. The eigenvalues, degrees of freedom and CPU time are shown in Table 2. The tolerance in Algorithm 4.1 is $\varepsilon=0.005$ and the total number of iterations in Algorithm 4.2 is 14.

We can see that the eigenvalues computed by Algorithm 4.1 and Algorithm 4.2 are very close to each other. As for the CPU time, Algorithm 4.2 saves about one fifth of Algorithm 4.1 indicating that the adaptive multigrid method is more efficient (see Fig. 3).

Table 2: The CPU time of elastic eigenvalue problem on L-shaped domain using Algorithm 4.1 and Algorithm 4.2.

\begin{tabular}{||c||rrc||rrc||}
\hline$l$ & Alg. 4.1 & DoF & CPU time & Alg. 4.2 & DoF & CPU time \\
\hline 2 & 55.4925 & 858 & 0.0887 & 55.4925 & 858 & 0.0700 \\
\hline 5 & 54.6566 & 3360 & 0.2646 & 54.6566 & 3360 & 0.2336 \\
\hline 8 & 54.4506 & 12032 & 0.9228 & 54.4506 & 12032 & 0.8044 \\
\hline 11 & 54.3914 & 41696 & 3.3549 & 54.3914 & 41696 & 2.9364 \\
\hline 14 & 54.3750 & 137938 & 11.9087 & 54.3750 & 137938 & 9.8909 \\
\hline
\end{tabular}




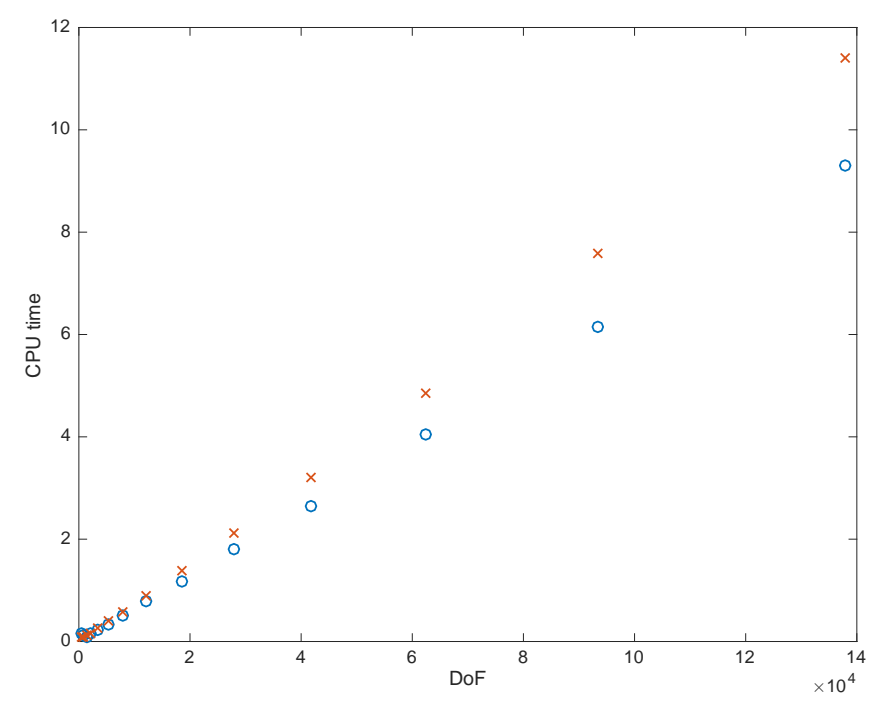

Figure 3: The CPU time on the L-shaped domain using Algorithm 4.1 and Algorithm 4.2. The circles and crosses stand for the results by using Algorithm 4.2 and Algorithm 4.1, respectively.

Example 5.3. The discontinuity of the Lamé parameters also leads to low regularity eigenfunctions. We consider another elastic eigenvalue problem with Lamé parameters being piecewise constant. Specifically, let the domain be the unit square $\Omega=[0,1] \times[0,1]$, $\rho \equiv 1, \bar{\lambda} \equiv 0$, and $\bar{\mu}=50$ on the top right corner $\Omega_{1}=(1 / 2,1] \times(1 / 2,1], \bar{\mu}=1$ on $\Omega \backslash \Omega_{1}$.

In Table 3 we show the computed eigenvalues, reference values, degrees of freedom, and convergence orders of Algorithm 4.1 and uniformly refined meshes. Here the size of initial mesh is $h \approx 0.1$, and the tolerance $\varepsilon=0.005$. For Algorithm 4.1, the total iterations of refinements is $l=23$, and the reference eigenvalues are computed in the same way as in Example 5.1. On the other hand, 5 times of uniform refinements are carried out. Due to the lack of knowledge of the regularity according to the first eigenfunction, we set the reference value for this method as the one for Algorithm 4.1. The log-log plot of the error

Table 3: The first elastic eigenvalue for discontinuous Lamé parameters using Algorithm 4.1 and uniformly refined mesh.

\begin{tabular}{||ccrc||ccrc||}
\hline$l$ & Algo. 1 & DoF & order & $l$ & uniform & DoF & order \\
\hline 3 & 47.1501 & 1206 & 0.9611 & 1 & 47.0355 & 1218 & - \\
\hline 7 & 46.5728 & 4648 & 0.8333 & 2 & 46.5948 & 5026 & 0.7844 \\
\hline 12 & 46.4249 & 21114 & 0.9126 & 3 & 46.4525 & 20418 & 0.7674 \\
\hline 17 & 46.3904 & 92660 & 0.9204 & 4 & 46.4060 & 82306 & 0.7533 \\
\hline 21 & 46.3825 & 295460 & 0.9588 & 5 & 46.3879 & 330498 & 0.7509 \\
\hline ref. & 46.3788 & & & ref. & 46.3788 & & \\
\hline
\end{tabular}




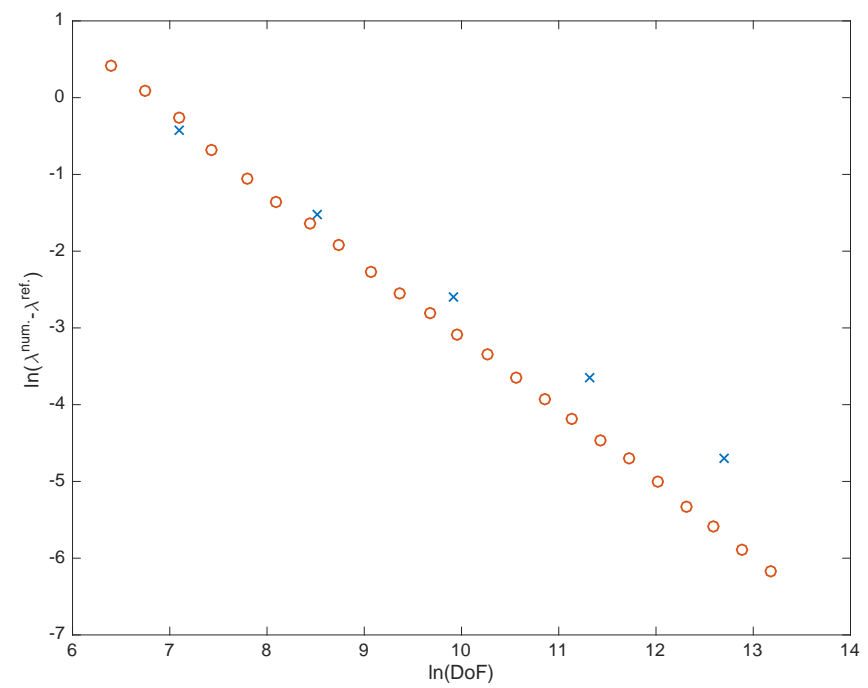

Figure 4: The difference between the first numerical eigenvalue and the reference value of the elastic eigenvalue problem with discontinuous Lamé parameters computed by Algorithm 4.1 and uniformly refined meshes. The circles and crosses stand for the results by using adaptively and uniformly refined meshes respectively.
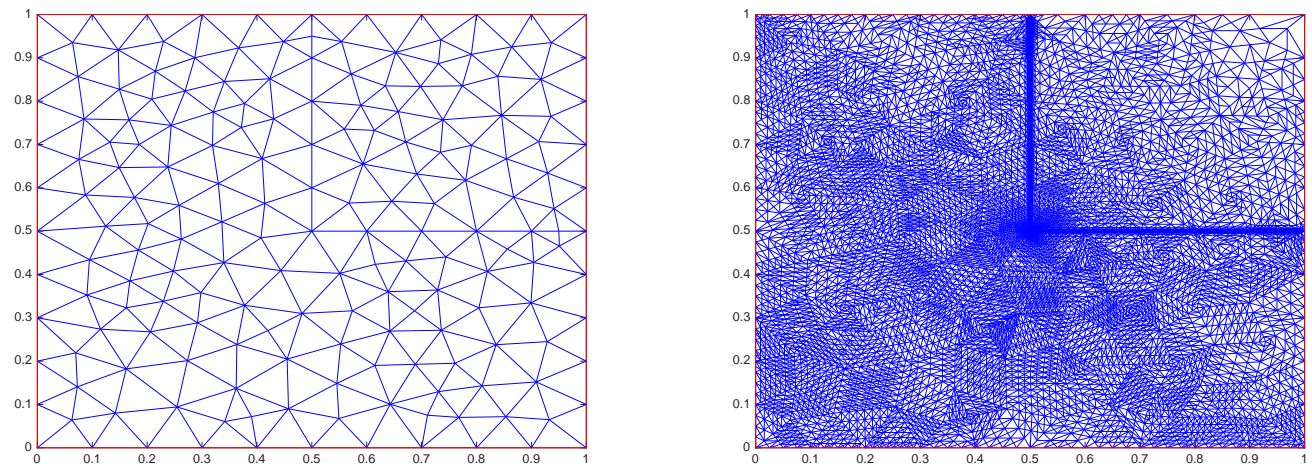

Figure 5: The meshes of Example 5.3, where the left is the initial mesh, and the right is the adaptive mesh at $l=12$.

against the degrees of freedom is shown in Fig. 4. The adaptive mesh at $l=12$ is shown in Fig. 5.

It can be seen that the convergence order of Algorithm 4.1 is close to the optimal order 1. In contrast, the uniform refinements leads to a low convergence order of approximately 0.7. In addition, from Fig. 4 we notice that the adaptive algorithm becomes more accurate after the 5th refinement. Fig. 5 shows that the adaptive method correctly catches the low regularity of the first eigenfunction across the line segments where the Lamé constants have a jump. 
Example 5.4. We solve the same problem as in Example 5.3 and compare the numerical results of Algorithm 4.1 and Algorithm 4.2. We set $\varepsilon=0.01$ in Algorithm 4.1. We show the computed eigenvalues, degrees of freedom and CPU time in Table 4 and Fig. 6. Similar results as Example 5.2 are observed. Algorithm 4.2 uses less computation time than Algorithm 4.1, which again confirms that the multigrid scheme is more efficient.

Table 4: The CPU time with discontinuous Lamé parameters using Algorithm 4.1 and Algorithm 4.2.

\begin{tabular}{||c||crr||crr||}
\hline$l$ & Algorithm 1 & DoF & CPU time & Algorithm 2 & DoF & CPU time \\
\hline 5 & 46.7261 & 2432 & 0.2038 & 46.7261 & 2432 & 0.1699 \\
\hline 9 & 46.4829 & 8732 & 0.6621 & 46.4829 & 8732 & 0.5658 \\
\hline 13 & 46.4140 & 28910 & 2.2211 & 46.4140 & 28910 & 1.8812 \\
\hline 17 & 46.3904 & 92660 & 7.4117 & 46.3904 & 92660 & 6.1783 \\
\hline 21 & 46.3825 & 295460 & 25.3496 & 46.3825 & 295460 & 20.5734 \\
\hline
\end{tabular}

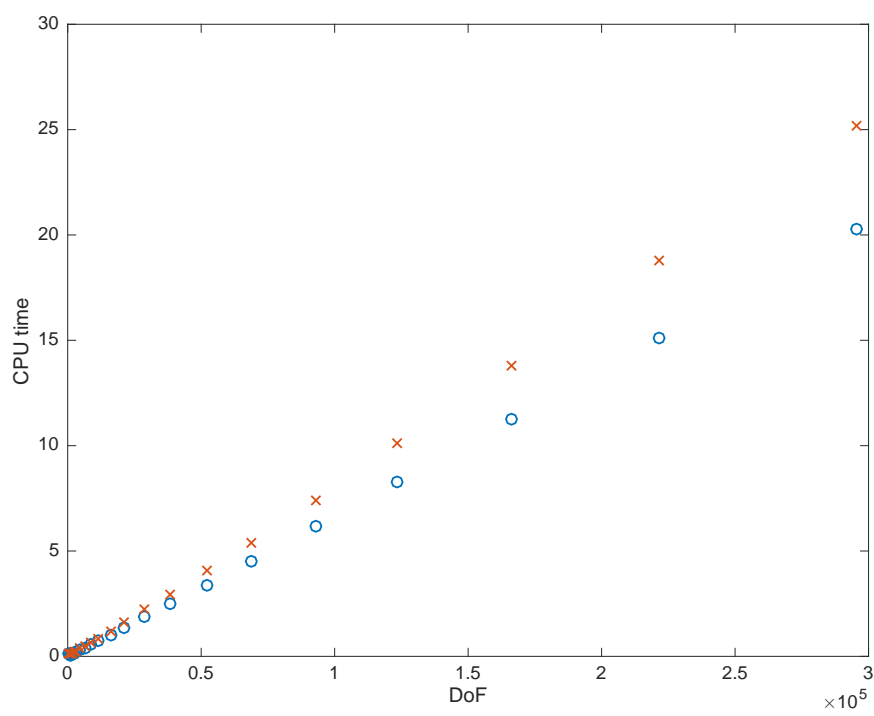

Figure 6: The CPU time for discontinuous Lamé parameters using Algorithm 4.1 and Algorithm 4.2. The circles and crosses stand for the results by using Algorithm 4.2 and Algorithm 4.1, respectively.

\section{Acknowledgments}

The research of J. Han was supported in part by the Doctoral Scientific Research Foundation of Guizhou Normal University (No. GZNUD[2018]33) and Guizhou Province Science and Technology Plan Project (No. [2018]5769). The research of Z. Zhang was supported in part by the National Natural Science Foundation of China under grants NSFC 
11471031, NSFC 91430216, NSAF U1530401, and the US National Science Foundation under grant DMS-1419040. The research of J. Sun was supported in part by National Science Foundation under grant DMS-1521555.

\section{References}

[1] I. Babuška and J. Osborn, Eigenvalue Problems, Handbook of Numerical Analysis, Vol. II, Finite Element Methods (Part 1), Edited by P.G. Ciarlet and J.L. Lions, Elsevier Science Publishers B.V. (North-Holland), 1991.

[2] D. Boffi, Finite element approximation of eigenvalue problems, Acta Numer. 19 (2010), 1-120.

[3] S.C. Brenner and L.R. Scott, The Mathematical Theory of Finite Element Methods, 3rd ed. New York, Spinger-Verlag, 2007.

[4] F. Chatelin, Spectral Approximations of Linear Operators, Academic Press, New York, 1983.

[5] P.G. Ciarlet, Basic error estimates for elliptic problems, in Finite element Methods (part 1), Handbook of Numerical Analysis, P. G. Ciarlet and J. L. Lions, Eds., vol. 3, Elsevier Science Publishers, North-Holand, 1991, 17-351.

[6] Ph. Clément, Approximation by finite element functions using local regularization, RAIRO Anal. Numer. 9 (1975), no. R-2, 77-84.

[7] X. Dai, L. He, and A. Zhou, Convergence and quasi-optimal complexity of adaptive finite element computations for multiple eigenvalues, IMA J. Numer. Anal. 35 (2015), no. 4, 1934-1977.

[8] X. Dai, J. Xu, and A. Zhou, Convergence and optimal complexity of adaptive finite element eigenvalue computations, Numer. Math. 110 (2008), no. 3, 313-355.

[9] A. Dello Russo, Eigenvalue approximation by mixed non-conforming finite element methods: the determination of the vibrational modes of a linear elastic solid, Calcolo 51 (2014), no. 4, 563-597.

[10] S. Domínguez, N. Nigam and J. Sun, Revisiting the Jones eigenproblem in fluid-structure interaction, submitted.

[11] J. Han, Z. Zhang, Y. Yang, A new adaptive mixed finite element method based on residual type a posterior error estimates for the Stokes eigenvalue problem, Numer. Methods Partial Differential Equations 31 (2015), no. 1, 31-53.

[12] V. Heuveline and R. Rannacher, A posteriori error control for finite element approximations of elliptic eigenvalue problems, Adv. Comput. Math. 15 (2001), no.1-4, 107-138.

[13] E. Hernández, Finite element approximation of the elasticity spectral problem on curved domains, J. Comput. Appl. Math. 225 (2009), no. 2, 452-458.

[14] X. Ji, J. Sun and H. Xie, A Multigrid Method for Helmholtz Transmission Eigenvalue Problems, J. Sci. Comput. 60 (2014), no. 2, 276-294.

[15] M.G. Larson, A posteriori and a priori error analysis for finite element approximations of selfadjoint elliptic eigenvalue problems, SIAM J. Numer. Anal. 38 (2000), no.2, 608-625.

[16] S. Lee, D.Y. Kwak, and I. Sim, Immersed finite element method for eigenvalue problems in elasticity, Adv. Appl. Math. Mech. 10 (2018), no. 2, 424-444.

[17] Q. Lin and H. Xie, A multi-level correction scheme for eigenvalue problems, Math. Comp. 84 (2015), no. 291, 71-88.

[18] S. Meddahi, D. Mora, and R. Rodríguez, Finite element spectral analysis for the mixed formulation of the elasticity equations, SIAM J. Numer. Anal. 51 (2013), no. 2, 1041-1063.

[19] E.E. Ovtchinnikov and L.S. Xanthis, Effective dimensional reduction algorithm for eigenvalue problems for thin elastic structures: a paradigm in three dimensions, Proc. Natl. Acad. Sci. USA 97 (2000), no. 3, 967-971. 
[20] J. Sun and A. Zhou, Finite element methods for eigenvalue problems. CRC Press, Taylor \& Francis Group, Boca Raton, London, New York, 2016.

[21] R. Verfürth, A Review of A Posteriori Error Estimation and Adaptive Mesh-Refinement Techniques, Wiley/Teubner, Stuttgart, 1996.

[22] T.F. Walsh, G.M. Reese, and U.L. Hetmaniuk, Explicit a posteriori error estimates for eigenvalue analysis of heterogeneous elastic structures, Comput. Methods Appl. Mech. Engrg. 196 (2007), no. 37-40, 3614-3623.

[23] H. Li and Y. Yang, Adaptive finite element method based on multi-scale discretizations for eigenvalue problems, Comput. Math. Appl. 65(2013), 1086-1102.

[24] J. Xu and A. Zhou, A two-grid discretization scheme for eigenvalue problems, Math. Comp. 70 (1999), no. 233, 17-25.

[25] Y. Yang and H. Bi, A two-grid discretization scheme based on shifted-inverse power method, SIAM J. Numer. Anal. 49 (2011), no. 4, 1602-1624.

[26] Y. Yang, H. Bi, J. Han, and Y. Yu, The shifted-inverse iteration based on the multigrid discretizations for eigenvalue problems, SIAM J. Sci. Comput. 37 (2015), no. 6, A2583-A2606. 